\title{
Utilisation de la thermographie IR pour l'estimation de l'évaporation régionale II. - Résultats obtenus à partir des données de satellite
}

\author{
Bernard SEGUIN $\left({ }^{*}\right)$, Suzanne BAELZ $\left({ }^{* *}\right)$, Jean-Marie MONGET $\left({ }^{* *}\right) \&$ Vincent PETIT $(* * *)$ \\ (*) I.N.R.A., Station de Bioclimatologie, Centre de Recherches d'Avignon, F84140 Montfavet. \\ (**) CTAMN, Ecole des Mines de Paris. Sophia Antipolis, F06500 Valbonne. \\ (***) Centre d'Informatique géologique. Ecole des Mines de Paris, F 77305 Fontainebleau.
}

RÉSUMÉ

Thermographie,

Infra-rouge thermique,

Télédétection par satellite,

Evaporation,

Echelle régionale.
Le traitement des données de satellite NOAA-5 et TIROS-N sur le site de la Crau a permis de suivre l'évolution des écarts entre la Crau sèche et la Crau irriguée au long de 3 années. Celle-ci fait apparaître une variation saisonnière liée au cycle du rayonnement solaire et des fluctuations d'amplitude en rapport avec l'intensité de la sécheresse. Les écarts constatés par rapport aux mesures au sol peuvent atteindre 5 à $6^{\circ}$, ce qui peut résulter de la non-prise en compte des effets de correction atmosphérique, mais également de la différence d'échelle spatiale concernée (50 à $100 \mathrm{~km}^{2}$ par satellite, quelques $\mathrm{m}^{2}$ au sol). L'analyse des données à l'échelle du « pixel » (environ $1 \mathrm{~km}^{2}$ ) n'améliore pas sensiblement les résultats, ce qui laisse supposer que les écarts constatés proviennent des passages entre l'échelle ponctuelle au sol et l'échelle du « pixel » du satellite. L'utilisation des relations établies dans la $1^{\mathrm{re}}$ partie de l'article permet d'établir une cartographie de l'ETR, dont la précision est sujette à caution par suite des différentes sources d'erreur analysées. Par contre, la thermographie IR par satellite semble plutôt bien adaptée, à l'heure actuelle, à l'estimation de la variation spatiale d'ETR.

\section{SUMMARY \\ Thermography, Thermal infrared, Satellite remote sensing, Evaporation Regional scale.}

Use of thermal I.R. for estimating regional evaporation. II. - Results obtained from satellite data.
Satellite data from NOAA-5 and TIROS-N on the Crau site were used to follow the time course of temperature differences between dry and irrigated zones during 3 consecutive years (fig. 3 ). A seasonal trend linked with solar incident radiation appears first, together with fluctuations related to dryness intensity. Comparison with simultaneous ground data (fig. 4) shows a general agreement, with deviations up to 5- $6^{\circ}$. These may result from atmospheric correction effects, but also from the large difference in scale (50 to $100 \mathrm{~km}^{2}$ from satellite - some $\mathrm{m}^{2}$ from ground measurements). The analysis of satellite data down to the pixel scale does not really give a better agreement (fig. 5), suggesting that the main source of deviations lies in the transition from local ground scale to the pixel scale.

The relationships established in the first part can be used to establish estimates of ET (fig. 6) which may then be mapped on a regional scale (fig. 7). Owing to the various sources of error found in the study, there is some doubt how successfully the method can be used to provide absolute values. However, thermal IR by satellite does seem very well adapted to the demonstration of spatial variation in ET.
L'analyse préliminaire décrite dans la première partie de cet article (SEGUIN et al., 1982) avait permis de délimiter 4 zones principales dans la plaine de la Crau. Ces zones ont fait l'objet d'un traitement systématique sur les données des satellites NOAA-5 et TIROS-N au CTAMN de Sophia Antipolis, à partir du prétraitement effectué à la Station de réception du CEMS (Centre d'Etudes en Météorologie Spatiale) de la Météorologie Nationale de Lannion. Les caractéristiques techniques des satellites, ainsi que de l'ensemble de la chaîne de traitement, sont discutées en détail dans la thèse de BAELz (1980). Un total de 99 passages a ainsi été analysé (27 pour 1977, 40 pour 1978 et 32 pour 1979). Quelques traitements du satellite HCMM ont également été effectués (d'une part au CTAMN de Sophia
Antipolis, d'autre part au CCR d'Ispra où a été testé le modèle Tellus mis au point par RosEMA et al., 1978) et font l'objet d'une autre publication (REINIGER et al., 1981).

\section{TRAITEMENT DES DONNÉES}

Pour chacune des 4 zones de la Crau, les histogrammes de fréquence des données thermiques sont obtenus à l'aide d'un traitement standard préalablement mis au point. Celuici comprend :

- une calibration en température de l'image,

- le filtrage isotrope des mesures, 


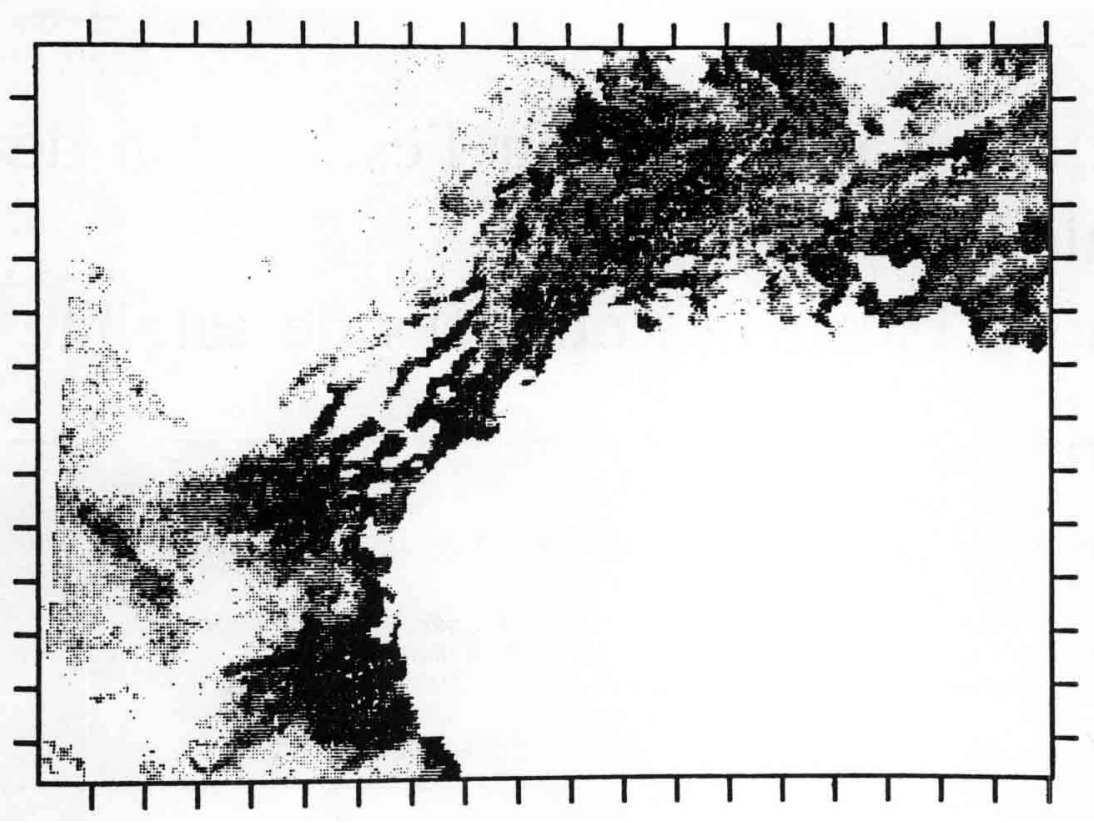

ITVETTAIRE ANTUEL DE LA TEMPERATURE DE LA CRAD BATELLITE NOAA5
DATE $=978$ HEURE TU $=837$ INDICE ETAT DE TRAITEMENT

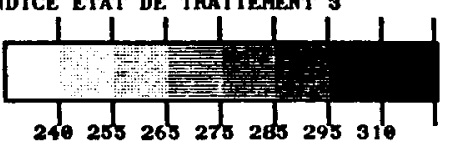

Figure 1

Visualisation de l'image après le $3^{e}$ traitement. View of satellite image after the third treatment.
— un filtrage vertical visant à éliminer le lignage des images,

- enfin une visualisation VERSATEK (imprimante traitement électrostatique en noir et blanc) (fig. 1).
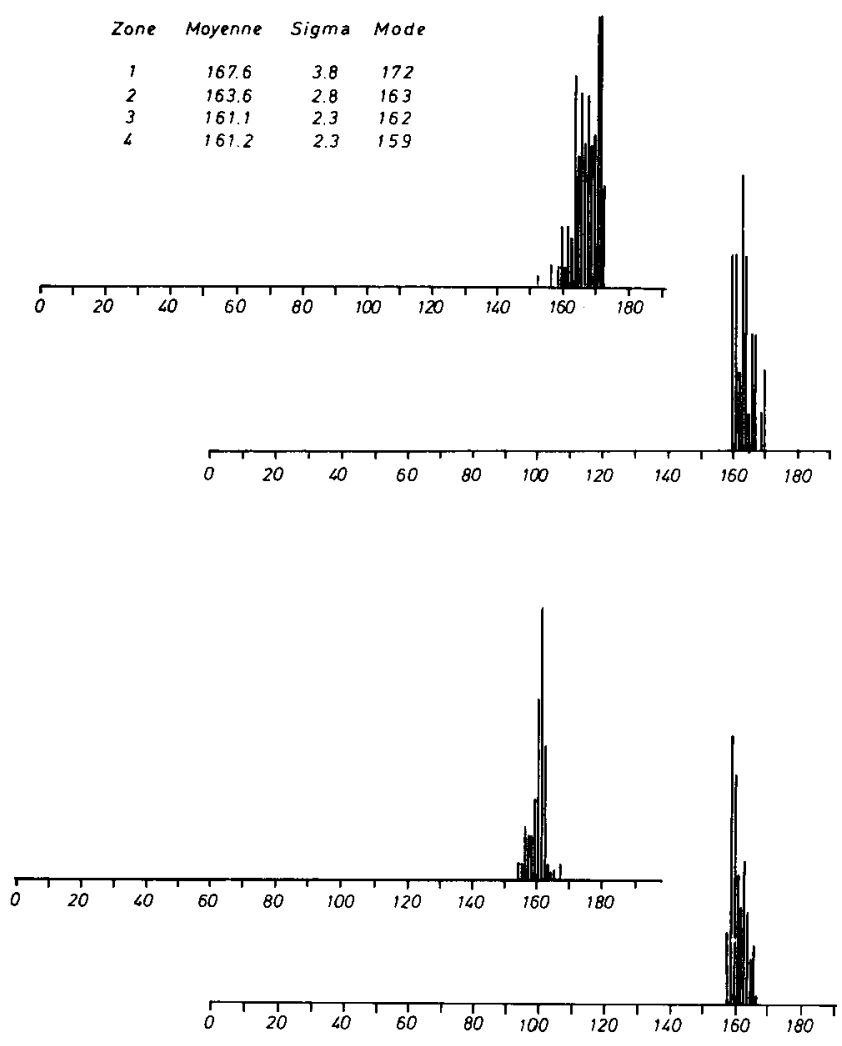

Figure 2

Histogrammes de température de surface pour chacune des 4 zones. Surface temperature histograms for the 4 Crau zones.

De plus, afin de pouvoir comparer numériquement les images obtenues à différentes dates, une image de référence a été définie sur laquelle ont été identifiés des amers. Chacune des images a été rectifiée par rapport à l'image de référence par superposition des amers, de façon à corriger la variation de l'orientation liée à la variation de l'orbite au moment de la prise de vue.

Les histogrammes thermiques ainsi obtenus (fig. 2) ont été utilisès :

- d'une part, pour effectuer une analyse de l'évolution dans le temps (années 1977 et 1978) des écarts de température de surface entre Crau sèche et zone irriguée,

- d'autre part, pour comparer (au cours de l'été 1978) les 2 séries de données (mesures au sol et données du satellite) chaque fois qu'elles étaient disponibles simultanément.

\section{II. ÉVOLUTION TEMPORELLE DES ÉCARTS THERMIQUES ENTRE CRAU SĖCHE ET CRAU IRRIGUÉE}

Le suivi des écarts de température entre les zones 2,3 et 4 de la Crau irriguée et la zone 1 de Crau sèche au cours des 3 années 1977,1978 et 1979 est présenté dans la figure 3.

Les écarts observés sont, de façon générale, plus faibles que ceux précédemment exposés à partir des mesures au sol, mais il faut noter que ces dernières étaient obtenues au voisinage du maximum de température (vers $13 \mathrm{~h} \mathrm{TU}$ ), alors que l'heure de passage du satellite se situait régulièrement vers $9 \mathrm{~h}$ TU.

L'examen de cette figure 3 fait apparaître d'une part, une variation saisonnière liée à l'intensité $d u$ rayonnement global, d'autre part, une amplitude estivale nettement plus élevée pour les années 1978 et 1979 que pour l'année 1977. Or, l'été 1977 avait été anormalement pluvieux pour la région (174 $\mathrm{mm}$ à Avignon pour juillet-août), au contraire de $1978(41 \mathrm{~mm})$ et $1979(72 \mathrm{~mm})$.

Par ailleurs, les écarts les plus importants apparaissent systématiquement pour la zone 3 , suivie par la zone 4 et ĺa zone 2 . Ce résultat est en bon accord avec le pourcentage de terres irriguées précédemment annoncé pour ces 3 zones (respectivement $70-80$ p. $100,50-60$ p. 100 et 40 p. 100).

$\begin{array}{ccrc}\text { Année } & \text { Zone 2 } & \text { Zone 3 } & \text { Zone 4 } \\ - & - & & - \\ 1977 & 3,5 & 8,0 & 4,5 \\ 1978 & 6,5 & 10,0 & 8,5 \\ 1979 & 7,0 & 9,0 & 7,0\end{array}$




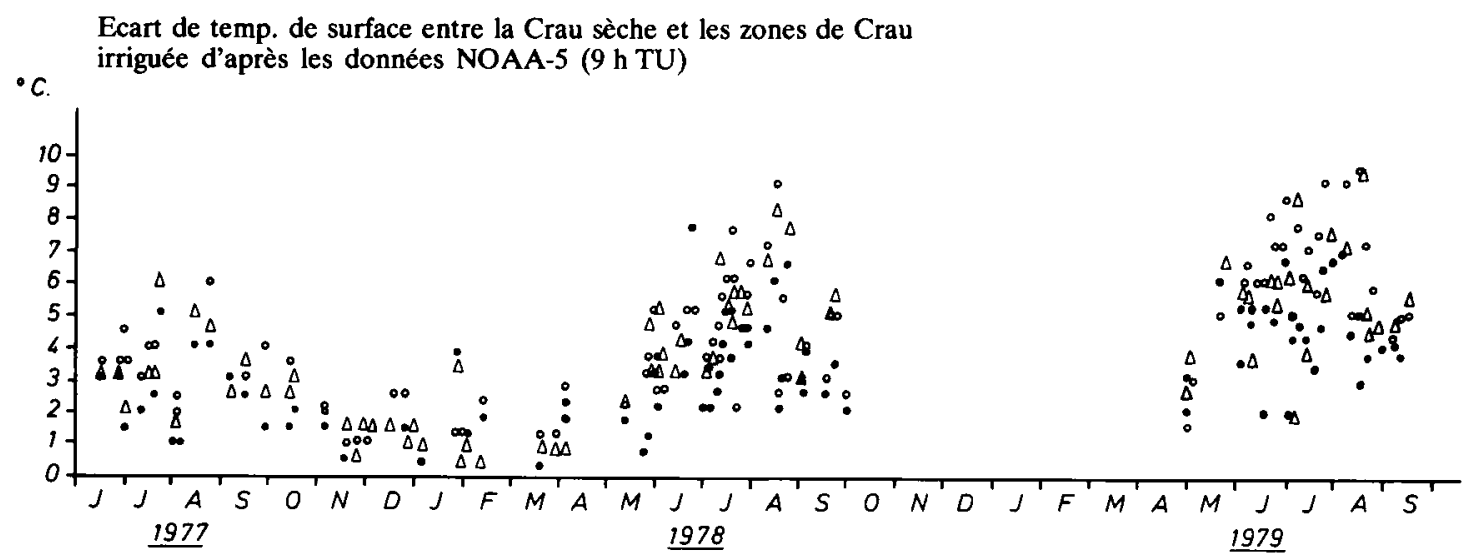

- Zone 2

- Zone 3

$\Delta$ Zone 4

Figure 3

Courbe de variation des écarts de température entre la Crau sèche et chacune des 3 zones de Crau irriguée au cours des années 1977, 1978, 1979.

Cette hiérarchie d'une part, entre les années, d'autre part, entre les zones, peut être exprimée en considérant le maximum d'écart constaté (mois d'août).

L'utilisation de la thermographie IR par satellite se révèle donc déjà, à ce niveau, comme un bon indicateur qualitatif du degré de sécheresse des surfaces concernées.

\section{COMPARAISONS ENTRE DONNÉES SATELLITE ET DONNÉES AU SOL}

La comparaison a été effectuée pendant la période la plus favorable, compte tenu de la disponibilité des mesures, à savoir le mois de juillet 1979 (fig. 4).

Cette comparaison fait apparaître globalement un accord satisfaisant, avec des écarts pouvant cependant atteindre 5 à $6^{\circ}$. Par ailleurs, un examen plus détaillé montre une surestimation par le satellite des températures plus basses de la Crau irriguée.

En dehors de l'hypothèse d'un étalonnage défectueux du radiomètre du satellite, les considérations suivantes peuvent être émises :

- l'absence de prise en compte de la correction atmosphérique peut conduire à des atténuations de l'ordre de 2 à $5^{\circ}$, sinon plus, ainsi que le montrent les calculs effectués à Ispra par REINIGER et al. (1981), ce qui pourrait justifier les écarts observés sur la Crau sèche.

- une correction du même ordre de grandeur devrait alors être effectuée sur les données de Crau irriguée. Leur surestimation par le satellite s'en trouverait renforcée. A ce niveau peut alors intervenir la différence de localisation spatiale entre les mesures au sol (de l'ordre du $\mathrm{m}^{2}$ ) et celles par satellite (de 100 ha à $100 \mathrm{~km}^{2}$ suivant le nombre de " pixcls " considéré).

Une étude spécifique a été entreprise pour évaluer l'influence de la dimension de la surface considérée sur la température moyenne observée. 3 échelles d'espace accessibles par satcllite ont été analysées : l'échelle ponctuelle du pixel, correspondant à la localisation des stations de mesure - l'échelle locale des 4 pixels entourant la parcelle de mesure - l'échelle moyenne de l'ensemble de la zone, à
Time course of thermal differences between dry Crau and each of the 3 zones of irrigated Crau in 1977, 1978 and 1979.

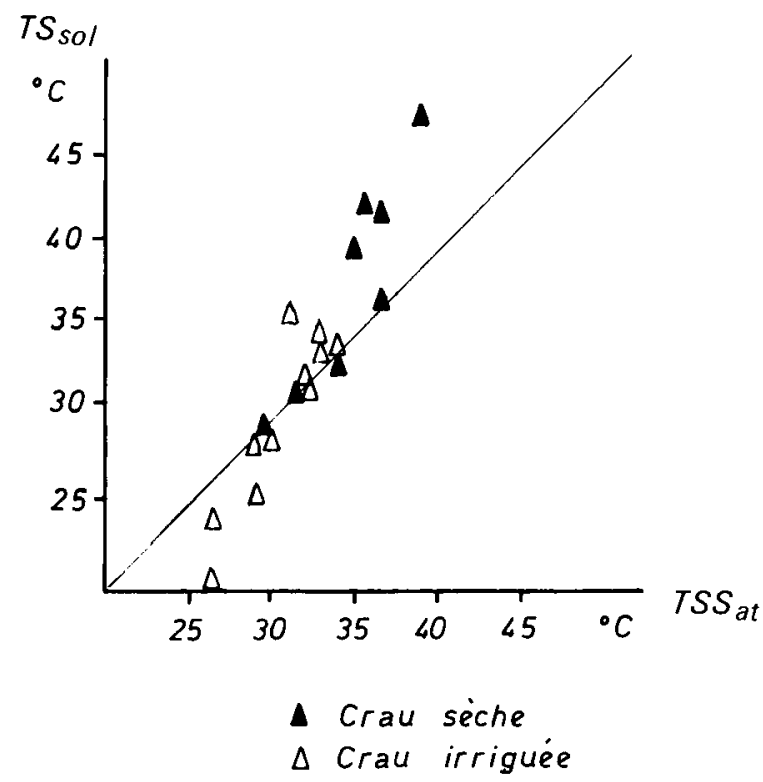

Figure 4

Comparaison des températures moyennes par satellite $\left(T_{S s}\right)$ et des températures enregistrées au sol $\left(T_{S s}\right)$ pendant le mois de juillet 1978.

Comparison of satellite mean values $\left(T_{S s}\right)$ and measured values on the ground $\left(T_{\mathrm{s}}\right)$ for surface temperature during july 1978.

partir de la décomposition des données «pixel» par " pixel» pour chaque zone (fig. 5).

Les résultats obtenus sont présentés de façon détaillée dans la thèse de BAELZ (1980). Si le passage à l'échelle locale des 4 pixels améliore très largement l'accord avec les températures du sol (de l'ordre de 0,5 à $1^{\circ}$ ), la prise en compte des données ponctuelles à l'échelle du pixel accentue, au contraire, les effets observés. On peut penser qu'à ce niveau de précision intervient de façon notable l'erreur de localisation du pixel unique et que l'on dépasse la limite de résolution spatiale du système, qui est donc optimale au niveau de 4 pixels.

Ce fait étant établi, le problème de l'écart avec les données au sol reste. entier. Plutôt qu'entre 500 ha et $100 \mathrm{~km}^{2}$ (gamme d'échelle sur laquelle joue la résolution du 


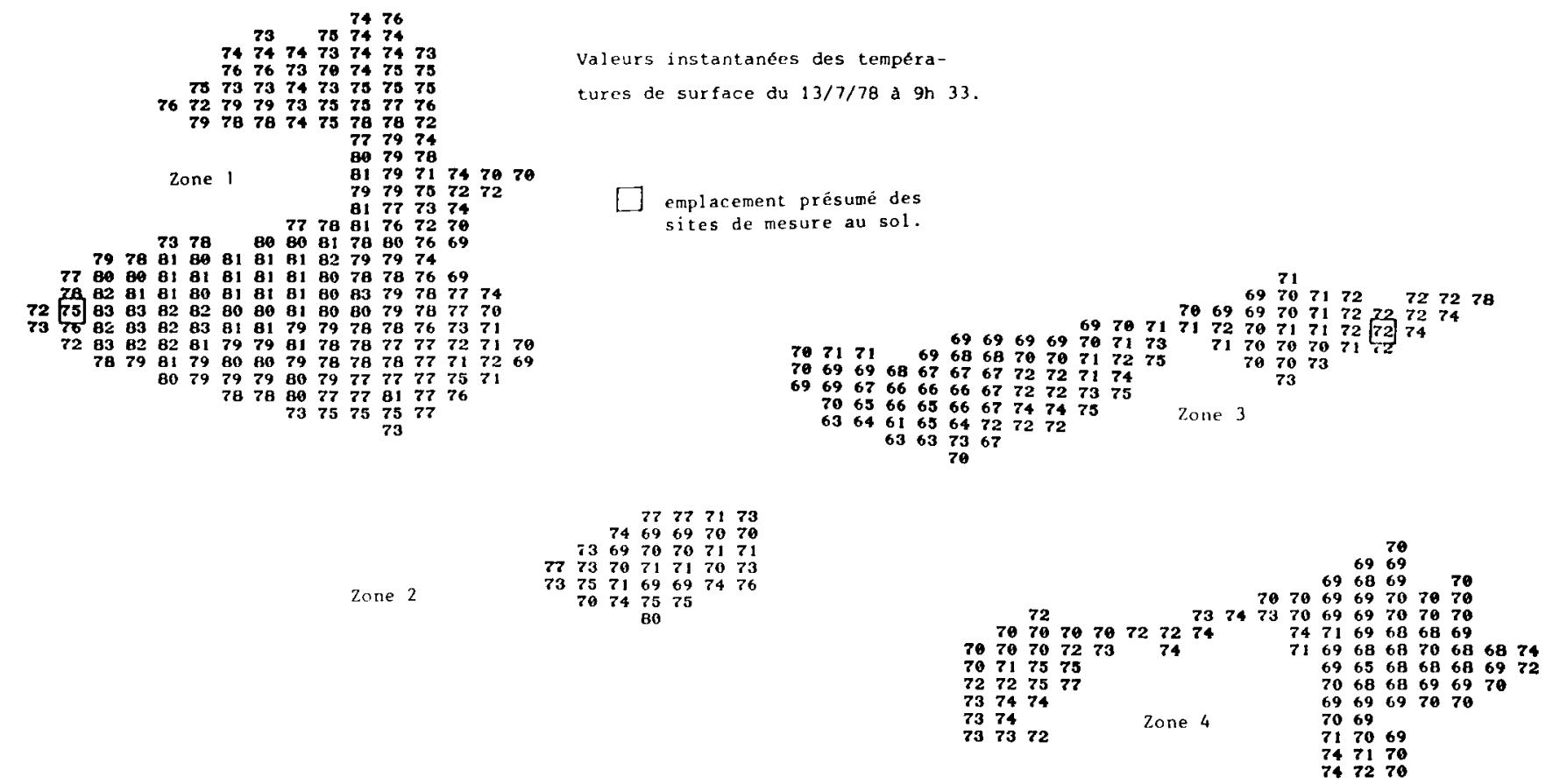

Figure 5

Répartition spatiale des températures de surface pour chacune des 4 zones de la Crau (les valeurs de température correspondent aux chiffres donnés divisés par le facteur 2).

Spatial variation of surface temperatures for each of the 4 zones of Crau (values given for each pixel have to be divided by 2 to be converted to temperature values).

satellite), c'est sur le passage entre l'échelle d'observation du radiomètre au sol (de l'ordre de $1 \mathrm{~m}^{2}$ ) et l'échelle du pixel que peut s'expliquer l'écart constaté. Il n'est pas illogique de supposer que la contribution de terrains non irrigués (haies, buissons, chemins, bâtiments d'exploitation, etc...) puisse expliquer une élévation de 2 à $5^{\circ}$ à l'échelle du pixel de la zone irriguée. Des mesures complémentaires sur cette gamme de résolution spatiale (par avion essentiellement) permettraient seules de pouvoir confirmer cette hypothèse.

\section{ESTIMATION DE L'ET INSTANTANÉE PAR SATELLITE}

A partir des mesures de bilan d'énergie effectuées au sol et de l'utilisation de la relation (1), il est possible d'obtenir des estimaticns de l'ET instantanée au moment du passage du satellite et de les comparer aux données de référence $\mathrm{ET}_{\mathrm{BE}}$ au sol disponibles au même instant. Le résultat obtenu pour les données disponibles en 1978 est présenté dans la figure 6.

Si l'accord est satisfaisant pour certains jours (essentiellement en zone irriguée), d'autres passages montrent des écarts considérables, principalement en zone sèche. S'il est donc possible de dresser des cartographies d'évaporation instantanée lorsque l'accord est satisfaisant (fig. 7) par exemple, il serait quelque peu illusoire d'en tirer systématiquement une cartographie quantitative de l'évaporation sur l'ensemble de la Crau, compte tenu des imprécisions constatées.

\section{CONCLUSION}

L'examen objectif des résultats concernés obtenus à la fois par l'expérimentation sur le terrain et par l'utilisation des satellites NOAA-5 et TIROS-N sur le site de la Crau conduit à un jugement nuancé, qui est un mélange de conclusions négatives et d'aspects positifs,

- l'estimation quantitative de l'ET de l'échelle locale à l'échelle régionale à partir de la thermographie IR par satellite peut difficilement améliorer, en valeur absolue, la précision d'évaluations simplifiées du type ET $=\frac{\Delta}{\Delta+\gamma} R_{n}$ ou $\mathrm{ET}=\mathrm{R}_{\mathrm{n}}$.

A ce niveau, l'utilisation de la relation (1) du bilan d'énergie apparaît plus comme une très bonne base de

$\mathrm{ET}_{\mathrm{BE}}$ : ET mesurée par la méthode du bilan d'énergie (rapport de BOWEN).

$\mathrm{ET}_{\mathrm{TS}}$-SAT : ET estimée à partir de la méthode du bilan d'énergie avec les données satellite.

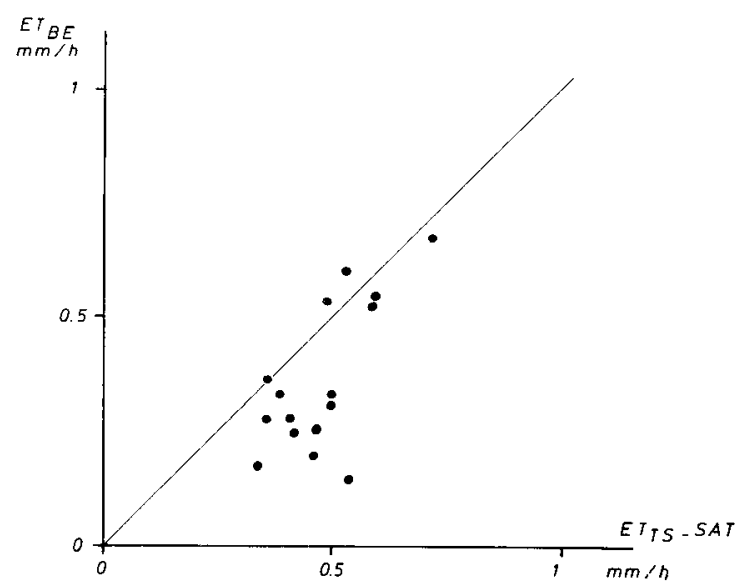

Figure 6

Corrélation entre $E T_{B E}$ et $E T_{T S}$ par satellite.

Correlation between $E T_{B E}$ measured on the ground and satellitederived $E T_{T S}$ values. 


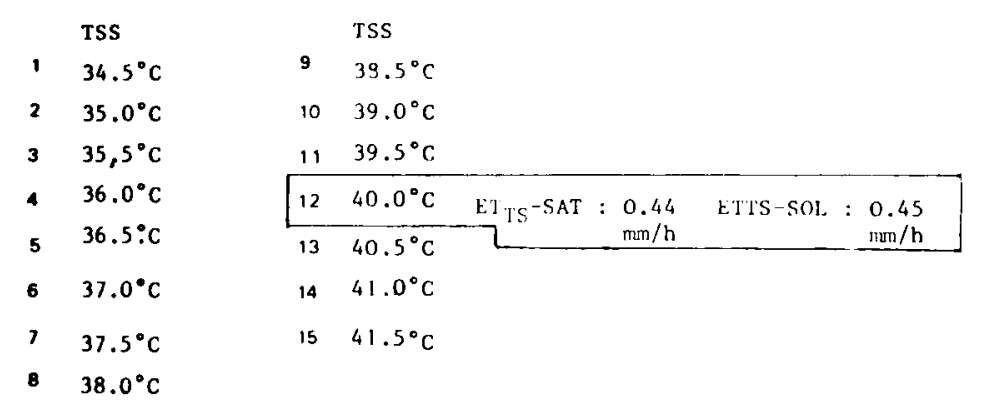

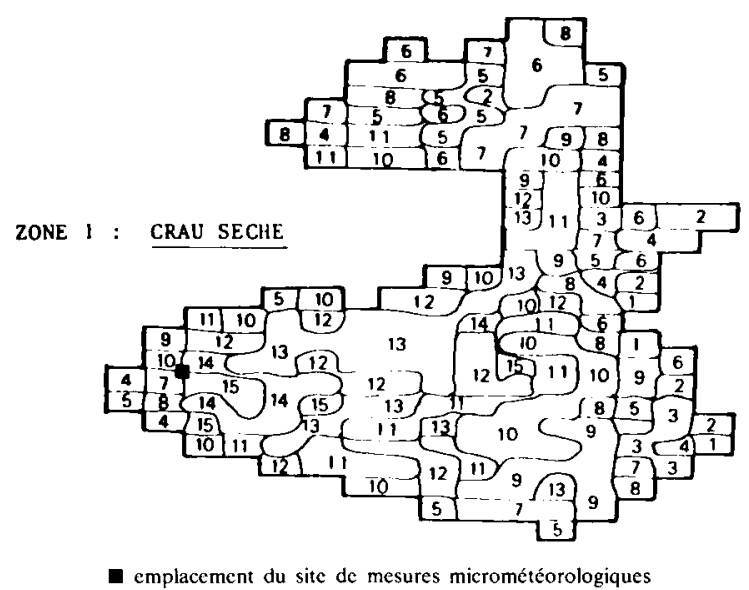

Figurc 7

Répartition spatiale de l'ET sur la région de la Crau sèche.

Spatial variation of ET within the dry Crau.

raisonnement sur la logique du phénomène que comme une formule d'estimation opérationnelle, essentiellement en raison du poids du paramètre $Z_{0}$ en conditions sèches. Par contre, et toujours au niveau opérationnel, l'utilisation de la relation dérivée de JACKSON et al. (1977) pourrait permettre une bonne approximation de l'ET à l'échelle de la décade ou du mois, avec des écarts individuels journaliers de l'ordre de $\pm 1,5 \mathrm{~mm} / \mathrm{j}$, à condition que :

- d'une part les valeurs numériques obtenues en Crau soient à peu près vérifiées pour d'autres types de surfaces et de climats,

- d'autre part la précision des mesures par satellite soit ramenée à un niveau acceptable de l'ordre de 1 à $2^{\circ}$, sans doute par la prise en compte systématique des corrections atmosphériques,

- en contrepartie de cet aspect plutôt négatif, des conclusions très positives peuvent être tirées de cette expérimentation en Crau pour ce qui concerne l'aspect de différences spatiales accessibles par la télédétection. Autant la fixation des valeurs absolues apparaît problématique, autant les variations dans l'espace du phénomène d'évaporation sont rendues qualitativement, et même quantitativement, de façon précise par la télédétection, ainsi que l'ont montré ITIER \& PERrier (1974). Celle-ci apparaît donc comme un outil nouveau, d'ores et déjà performant, pour rendre compte aussi bien de la localisation et la délimitation des surfaces que de leurs contrastes hydriques.

Plutôt que d'envisager donc, à l'heure actuelle, l'utilisation directe de la thermographie IR pour le calcul de l'évaporation, il semble plus rationnel de considérer les satellites comme porteurs d'une information inédite sur la variation spatiale du bilan hydrique. Dans cette optique, l'estimation de l'évaporation pourrait passer par la combinaison de modèles de bilans hydriques - par exemple BAiER (1980) ou Cholsnel (1977) - et de passages de satellite permettant de cartographier l'extension spatiale des paramètres significatifs de ces modèles (en premier lieu la réserve utile RU au sol). Dans l'attente d'un progrès technologique permettant d'améliorer l'aspect d'extrapolation temporelle (satellites géostationnaires à résolution spatiale améliorée, ou 2 à 3 passages quotidiens de satellites combinés, sans compter l'extension des mesures radar), c'est essentiellement l'aspect d'extrapolation spatiale qui nous paraît pouvoir aujourd'hui être retiré de la thermographie IR. Les recherches s'orientent donc plutôt vers la cartographie des unités de sols considérées sous l'angle du bilan hydrique.

Reçu le 15 avril 1981. Accepté le 14 octobre 1981.

\section{RÉFÉRENCES BIBLIOGRAPHIQUES}

Baelz S., 1980. Utilisation de thermographies infra-rouge recueillies par satellite pour le calcul de l'évapotranspiration à l'échelle régionale. Thèse de Doctorat de $3^{\mathrm{c}}$ cycle. Université de Paris VI. Sciences de l'Eau. $119 \mathrm{p}$.

Baier W., 1980. Water valance in crop-yicld models. In * Application of remote sensing to agricultural production forescasting". Proceedings du Séminaire d'lspra (Italie). A. BERG Ed., 119-131. Publié par BALKEMA A. A. Rotterdam.

Choisnel E., 1977. Le bilan d'énergie et le bilan hydrique du sol. $L a$ Météorologie $\mathrm{VI}^{\mathrm{c}}$ série, $\mathrm{n}^{\mathrm{o}} 11$. Numéro Spécial sur l'évapotranspiration, 103-159.

Itier B., Perrier A., 1974. Thermographie et détermination de l'ETR des surfaces naturelles. Comm. au $1^{\text {er }}$ Congrès Européen de Thermographie Amsterdam, Juin 1974. Note interne I.N.R.A. Station de Bioclimatologie, Versailles, $26 \mathrm{pp}$.
Jackson R. D., Reginato R. J., Idso S. B., 1977. Wheat canopy temperature: a practical tool for evaluating water requirements. Water Resour. Res., 13 (3), 651-656.

Reiniger P., Huygen J., Megier J., Seguin B., 1981. Estimates of regional ET in South-eastern France using thermal and albedo data from the HCMM satellite. Comm. pres. au Symposium ERIM "Remote Sensing of Arid and Semi-arid lands". Le Caire (Egypte). 3-9 Nov. 1981.

Rosema A., Bijleveld J. H., Reiniger P., Tassone G., Blyth K., Gurney R. J., 1978. « Tell-us », a combined surface temperature, soil moisture and evaporation mapping. 12th Int. Symp. Remote Sensing of Environ. Manille, Philippines, Avril 1978.

Seguin B., Baelz S., Monget J. M., Petit V., 1982. Utilisation de la thermographie IR pour l'estimation de l'évaporation régionale. I. Mise au point méthodologique sur le site de la Crau. Agronomie, 2 (1), 7-16. 